\title{
Finding the Best Not the Most: Regularized Loss Minimization Subgraph Selection for Graph Classification
}

\author{
Shirui Pan, ${ }^{\mathrm{a}, *}$, Jia $\mathrm{Wu}^{\mathrm{a}}$, Xingquan Zhu ${ }^{\mathrm{b}}$, Guodong Longa ${ }^{\mathrm{a}}$, Chengqi Zhang ${ }^{\mathrm{a}}$ \\ ${ }^{a}$ Centre for Quantum Computation \& Intelligent Systems, FEIT, University of Technology Sydney, Australia \\ ${ }^{b}$ Department of Computer and Electrical Engineering \& Computer Science, Florida Atlantic University, \\ USA
}

\begin{abstract}
Classification on structure data, such as graphs, has drawn wide interest in recent years. Due to the lack of explicit features to represent graphs for training classification models, extensive studies have been focused on extracting the most discriminative subgraphs features from the training graph dataset to transfer graphs into vector data. However, such filter-based methods suffer from two major disadvantages: (1) the subgraph feature selection is separated from the model learning process, so the selected most discriminative subgraphs may not best fit the subsequent learning model, resulting in deteriorated classification results; (2) all these methods rely on users to specify the number of subgraph features $K$, and suboptimally specified $K$ values often result in significantly reduced classification accuracy.

In this paper, we propose a new graph classification paradigm which overcomes the above disadvantages by formulating subgraph feature selection as learning a $K$ dimensional feature space from an implicit and large subgraph space, with the optimal $K$ value being automatically determined. To achieve the goal, we propose a regularized loss minimization-driven (RLMD) feature selection method for graph classification. RLMD integrates subgraph selection and model learning into a unified framework to find discriminative subgraphs with guaranteed minimum loss w.r.t. the objective func-
\end{abstract}

\footnotetext{
${ }^{*}$ Corresponding author. Address: Centre for Quantum Computation \& Intelligent Systems, FEIT, University of Technology, Sydney, Ultimo, NSW 2007, Australia. Tel: +61 450768511. Fax: +61 29514 4535.

Email addresses: shirui.pan@uts.edu.au (Shirui Pan), jia.wu@student.uts.edu.au (Jia Wu), xzhu3 fau. edu (Xingquan Zhu), guodong. long@uts . edu . au (Guodong Long), chengqi. zhang@uts. edu. au (Chengqi Zhang)
} 
tion. To automatically determine the optimal number of subgraphs $K$ from the exponentially large subgraph space, an effective elastic net and a subgradient method are proposed to derive the stopping criterion, so that $K$ can be automatically obtained once RLMD converges. The proposed RLMD method enjoys gratifying property including proved convergence and applicability to various loss functions. Experimental results on real-life graph datasets demonstrate significant performance gain.

Keywords: Feature Selection, Classification, Graph Classification, Sparse Learning

\section{Introduction}

Recent years have witnessed an increasing number of applications involving objects with structural relationships, including chemical compounds in Bioinformatics [1], brain networks [2], image structures [3], and academic citation networks [4]. For these applications, graph is a natural and powerful tool for modeling and capturing dependency relationships between objects.

Unlike conventional data, where each instance is represented in a feature-value vector format, graphs exhibit node-edge structural relationships and have no natural vector representation ${ }^{1}$. As a result, a common practice is to transfer graphs into vectors $[5,6,7,8,9]$ in structure space or in Euclidean space, so that traditional machine learning algorithms such as Support Vector Machines (SVM) and Decision Tree can be applied. In the structure space (also referred to as quotient space) [7, 8], the distance relations and nature of the original data are preserved, and some geometrical and analytical concepts such as derivatives of functions on structures can be determined, so that it can be applied to solve problems in structural pattern recognition. In the Euclidean space, the structural relations may be lost, but it provides simpler and more powerful analytical techniques for data analysis. Therefore, numerous approaches $[10,9,11,12,13,14,15,16,17,18]$ have been proposed to represent graphs in Euclidean space. The key idea of transferring graphs into vectors in Euclidean space is to extract a set of subgraphs as features and use the presence/absence of the features

\footnotetext{
${ }^{1}$ In this paper, we only consider graphs with labels but no other feature values on nodes and edges.
} 


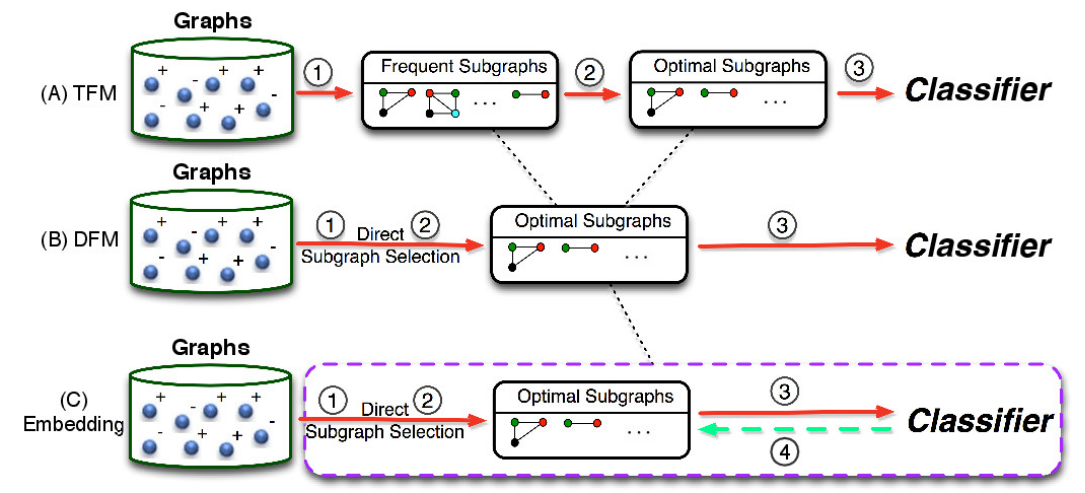

Figure 1: Subgraph-based methods for graph classification from the feature selection perspective. TFM methods (A) sequentially perform frequent subgraph mining (1), optimal feature selection (2), and classifier learning process (3). DFM methods (B) integrate the feature selection (2) into the frequent subgraph mining (1) process. Our embedding method RLMD (C) unifies all steps (1)(2)(3) into a whole framework, and iterates until convergence (4).

to represent each graph. From a feature selection perspective [19], these subgraphbased algorithms follow a filter approach for graph classification, i.e., the subgraph feature selection and the subsequent model training are separated into two steps. In summary, existing filter-based graph classification methods roughly fall into the following two categories:

Two-step Filter Methods (TFMs): This type of method first mines a set of frequent subgraphs as features and then applies a feature selection procedure to the discovered subgraphs, and uses the selected subgraph features to learn a classifier (e.g., an SVM or Naive Bayes), as shown in Fig. 1 (A). An early study [9] has shown that using frequent subgraphs as features can achieve reasonable good classification results. However, because TFMs separate subgraph feature discovery and feature evaluation into two steps, they may suffer from severe disadvantage in that the number of discovered subgraphs will grow exponentially when the minimum support value for subgraph mining is low. As a result, it will make the feature selection step heavily time-consuming. On the other hand, for relatively high minimum support values, many good subgraphs are pruned out because they do not meet the frequency requirement, so cannot be found to represent graphs. 
Direct Filter Methods (DFMs): To improve the subgraph feature selection efficiency, numerous approaches $[11,12,15,16,17]$ have been proposed to combine subgraph mining and feature selection into one step, representing a direct discriminative feature selection [18] scheme. So the feature selection is integrated into a subgraph mining process (Fig. 1 (B)), with pruning rules derived from the anti-monotone property of the significance ( $p$-value) of each graph being used to reduce the search space. While DFMs substantially overcome the subgraph feature selection bottleneck, they also have a number of major disadvantages: (1) The subgraph selection is separated from the model learning process, so the selected subgraphs features may not best fit the underlying learning model, and (2) All these methods require users to specify the number of subgraph features $K$, whereas the optimal number of subgraphs $K$ required for training a good classifier for graph classification is unknown and difficult to determine. Although subgraphs are selected using optimized measures, due to the redundancy inside the feature set, the accuracy of the classifiers, when varying the number of selected subgraph features $K$, is highly variable, as shown in Fig. 2. This is a common problem for all existing filter-based graph classification methods.

The above observations motivate the proposed research which aims to integrate subgraph mining, feature selection, and model training into one single framework (Fig. 1 (C)) with the optimal number of subgraphs $K$ being automatically determined for graph classification. To achieve this goal, we formulate subgraph feature selection as the problem of learning a $K$-dimensional feature space from a huge subgraph space in order to result in minimum regularized loss on the training data as follows:

$$
\min _{\boldsymbol{w}} \frac{1}{n} \sum_{i=1}^{n} \mathcal{L}\left(y_{i}, f\left(\boldsymbol{x}_{i}\right)\right)+\gamma R(\boldsymbol{w})
$$

where $\left\{\boldsymbol{x}_{i}, \cdots, \boldsymbol{x}_{n}\right\}$ are the vector representations of the training graphs, $\mathcal{L}$ is a loss function measuring the difference between the prediction $f\left(\boldsymbol{x}_{i}\right)$ and the true label $y_{i}$, and $R(w)$ is a regularization term on parameters $w$ to avoid over-fitting.

Indeed, the optimization in Eq.(1) has been widely studied [20,21, 22] in machine learning community, but mainly for data with vector format. Several significant challenges remain for graph data:

1. Implicit Subgraph Features: For graph classification, no subgraph features are 
readily available (i.e., $\boldsymbol{x}_{i}$ is unknown) for training the model in Eq.(1). Instead, the feature space used to represent graphs is implicit and needs to be discovered by subgraph mining procedure as needed.

2. $K$-dimensional Features from Huge Subgraph Space: The number of subgraph candidates representing graphs is exponentially large. Finding an optimal number of $K$ subgraphs for different graph datasets (in order to result in best classifiers), is crucial but has not been addressed by existing research.

In this paper, we propose a unified regularized loss minimization-driven (RLMD) graph classification framework. Our theme is to progressively select the most discriminative subgraph features from the training data in order to achieve minimum regularized loss for a well defined objective function. To integrate subgraph selection into the model learning process (Challenge 1), we formulate an objective function and design a subgradient method to induce a measurement to assess the utility of each subgraph, so that the best subgraph features can be identified and incrementally included to optimize the objective function for maximum performance gain. To determine the optimal $K$ value for each dataset (Challenge 2), we use an elastic net [21] and derive a stopping condition, so that the $K$ value can be automatically obtained when the algorithm converges. By using the automatically determined optimal $K$ value, as shown in Fig. 2, RLMD finds 180 best subgraphs and achieves the best performance, which is $6 \%$ more accurate than the second best method.

The main contributions of this paper are summarized as follows:

- We propose an embedded and theoretically convergent graph classification algorithm, which can automatically determine the optimal number of subgraphs $K$ for graph classification. This is a unified approach in the sense that (1) it can employ any differentiable loss function (including least squares, exponential, and logistic loss functions) for graph classification; and (2) it integrates subgraph mining, feature selection, and model learning into one single framework.

- We generalize the column generation technique of gBoost [23] for graph classification, and demonstrate that gBoost [23] is a special case of our loss minimization algorithm. 


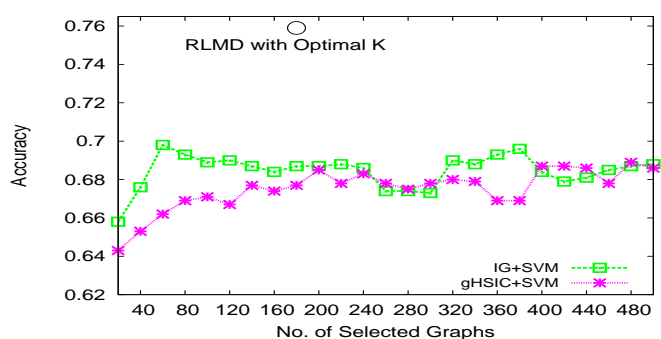

Figure 2: Classification accuracy for filter subgraph-based methods w.r.t. different numbers of subgraphs on the NCI-1 chemical compound dataset. IG is a TFM method which uses information gain to select subgraphs, whereas gHSIC [12] is a DFM method. All methods use SVM as a base classifier. The optimal number of subgraph features $K$ is crucial, but difficult to decide for filter methods. In comparison, the proposed method (RLMD) automatically finds 180 best subgraphs and achieves the highest accuracy, which is $6 \%$ more accurate than the second best method.

- We propose the use of elastic net (which integrates two sparsity-inducing regularization norms, $\ell_{1}$-norm and $\ell_{2}$-norm) to produce a sparse and robust solution for discriminative subgraph selection.

- We derive a branch-and-bound rule according to the subgradient of our objective function to prune search space for optimal subgraph mining.

- Experimental results show that our algorithm RLMD outperforms two-step filter methods (TFMs), direct filter methods (DFMs), and gBoost algorithm with significant performance gain.

\section{Related Work}

Our work is closely related to graph-based learning and graph classification.

\subsection{Graph-based learning}

Learning from data with dependency structures has been commonly addressed by existing research. Instead of considering samples as I.I.D observations, graph-based learning takes the relationships/correlations between samples to ensure effective learning. For example, graph-based approaches have been popularly used to propagate labels in semi-supervised learning $[24,25,26]$, where training samples are connected through one or multiple graphs. A recent method [27] considers preserving global and 
local structures inside the training data for feature selection. For large scale networks, predicting linkage relationships between nodes (i.e. link prediction) can be used for friendship recommendation in social networks [28], or suggesting potential interactions between proteins in bioinformatics research. A recent work [29] proposed to use latent feature kernels to support link prediction on sparse graphs. All above methods consider a large scale network with thousands (or millions) of integer-connected nodes in the network. In contrast, we consider small graph classification problem, in which each graph has a label indicating the property of the graph, and the graph normally contains tens or several hundreds of nodes. The purpose is to predict the label of the graph by using node and structure information inside the graphs, for purposes such as chemical compound activity prediction [1] and gender classification using magnetic resonance connectome (i.e. brain-graph) [2].

\subsection{Graph Classification}

Existing methods for graph classification $[18,10,9,11,12,13,14,15,16,17$, $23,30,31]$ can be roughly categorized into two groups: similarity-based methods and vector representation-based methods.

\subsubsection{Similarity-based methods}

These approaches aim to directly learn global similarities between graphs by using graph kernels [9, 32, 33, 34] or graph embedding [35]. Global similarities are then fed to similarity-based classifiers, such as KNN or SVM, for learning. One clear drawback of global similarity-based approaches is that the similarity is calculated based on global graph structures, such as random walks or embedding space. Therefore, it is not clear which substructures are more important for classifying graphs into different classes.

\subsubsection{Vector representation-based methods}

Another branch of methods transfer graphs into vector representations in structure space or in Euclidean space. In structure space [7, 8], geometrical and analytical concepts such as the angle between structures and the derivatives of functions on structures can be obtained, so that the structural pattern recognition problems can be formulated as optimization problems with certain cost functions. In Euclidean space, the goal is 
to transfer graphs into vector representations in Euclidean space so existing analytical techniques can be applied for data analysis. Methods in this category are mainly filterbased approaches, including two-step filter methods (TFMs) or direct filter methods (DFMs).

TFMs are straightforward approaches for graph classification which simply decompose frequent subgraph generation and selection as two separated steps. An early work [9] has shown that learning an SVM classifier based on the discovered frequent subgraphs can achieve reasonably good accuracy for graph classification. On the other hand, research $[16,15]$ also indicates that TFM methods may result in a bottleneck for the subsequent feature selection module. Specifically, the number of frequent subgraphs will grow exponentially if the minimum support threshold is low, which imposes a great challenge for the subsequent feature selection task. This challenge has motivated many direct filter methods (DFMs), which seek to integrate subgraph discovery and feature selection into one step.

For DFMs (a review on this category can be found in [18]), a key issue is to define a proper measurement to assess the utility of each subgraph. Yan et al [17] proposed a LEAP algorithm to exploit the correlation between structural similarity and significance similarity, so that a branch-and-bound rule can be derived to prune out unpromising searching space efficiently. Ranu and Singh [16] proposed a scalable GraphSig algorithm, which is able to mine significant subgraphs with low frequencies. Thoma et al. [15] propose a CORK algorithm to find subgraph features. Recently, researchers have extended DFM to other graph applications, and have proposed effective algorithms such as gSemi [11] for the semi-supervised setting, gCGVFL [36] for multiview learning, gHSIC [12] for multi-label classification, and our recent multi-graph classification for classifying graph bags, each containing multiple graphs [37, 38].

Although filter methods for graph classification have been extensively studied, they all suffer from two major disadvantages: (1) the feature selection is not linked to the model learning process. As a result, the selected subgraph features may not best fit the underlying learning algorithms; and (2) the optimal number of subgraphs $K$ for graph classification is difficult to decide and often varies from dataset to dataset, and inappropriately specified $K$ value often results in significantly reduced classification 
accuracy. This is the common drawback for filter-based methods [19].

Embedded Methods. Our algorithm belongs to the embedded approach which integrates the subgraph selection into the model training process. In this subcategory, Saigo et al [23] proposed a gBoost (its variants for imbalanced graph classification [39, 40] and cost-sensitive learning [41] are proposed recently) algorithm which formulates the graph classification as a linear program. As will be elaborated in Sec 4.6, our algorithm is more general in the sense that it can adopt any differentiable loss function and use more robust regularization to produce better performance. In fact, gBoost [23] can be considered as a special case of our loss minimization problem.

\section{Problem Definition}

Definition 1. Connected Graph: A graph is denoted by $G=\left(\mathcal{V}, E, L=\left\{L_{1}, L_{2}\right\}, \mathcal{A}=\right.$ $\left.\left\{\mathcal{A}_{1}, \mathcal{A}_{2}\right\}\right)$, where $\mathcal{V}$ is the vertex set, $E \subseteq \mathcal{V} \times \mathcal{V}$ is the edge set, $\mathcal{A}=\left\{\mathcal{A}_{1}, \mathcal{A}_{2}\right\}$ with $\mathcal{A}_{1}$ and $\mathcal{A}_{2}$ being the set of labels for vertices and edges, respectively; and $L=\left\{L_{1}, L_{2}\right\}$, $L_{1}: \mathcal{V} \rightarrow \mathcal{A}_{1}, L_{2}: E \rightarrow \mathcal{A}_{2}$ are labeling functions that assigns labels to a node or an edge, respectively. A connected graph is a graph such that there is a path between any pair of vertices.

In this paper, we focus on connected graphs and assume that each graph $G$ has a class label $y, y \in \mathcal{Y}=\{-1,+1\}$. We only focus on binary-class classification tasks, but our methods can be easily extended to multi-class tasks.

Definition 2. Subgraph: Given two graphs $G=\left(\mathcal{V}, E, L=\left\{L_{1}, L_{2}\right\}, \mathcal{A}=\left\{\mathcal{A}_{1}, \mathcal{A}_{2}\right\}\right)$ and $g_{k}=\left(\mathcal{V}^{\prime}, E^{\prime}, L^{\prime}=\left\{L_{1}^{\prime}, L_{2}^{\prime}\right\}, \mathcal{A}^{\prime}=\left\{\mathcal{A}_{1}^{\prime}, \mathcal{A}_{2}^{\prime}\right\}\right), g_{k}$ is a subgraph of $G\left(\right.$ i.e., $\left.g_{k} \subseteq G\right)$ if there is an injective function $\hat{f}: \mathcal{V}^{\prime} \rightarrow \mathcal{V}$, such that $\forall(a, b) \in E^{\prime}$, we have $(\hat{f}(a), \hat{f}(b)) \in$ $E, L_{1}^{\prime}(a)=L_{1}(\hat{f}(a)), L_{1}^{\prime}(b)=L_{1}(\hat{f}(b)), L_{2}^{\prime}(a, b)=L_{2}(\hat{f}(a), \hat{f}(b))$. If $g_{k}$ is a subgraph of $G\left(g_{k} \subseteq G\right), G$ is a supergraph of $g_{k}\left(G \supseteq g_{k}\right)$.

Subgraph-based Graph Classification: Given a set of labeled graphs $\mathcal{T}=\left\{\left(G_{1}, y_{1}\right)\right.$, $\left.\cdots,\left(G_{n}, y_{n}\right)\right\}$, subgraph-based graph classification aims to select an optimal set of discriminative subgraphs $\mathcal{F}_{1}$ from $\mathcal{T}$, and learn a classification model from the reduced subgraph space $\mathcal{F}_{1}$ to predict previously unseen test graphs with a maximum accuracy. Set $\mathcal{F}_{1}$ is optimal if the classifier learned from $\mathcal{F}_{1}$ has the highest classification accuracy, 
compared to classifiers trained from any subset of $\mathcal{T}$. A major feature of our method is that it can automatically determine the best set of subgraph features to represent each graph datasets without requiring users to specify the number of subgraph features. This essentially advances the existing subgraph feature-based graph classification methods from finding the most discriminative subgraph features to finding the best subgraph set for maximum accuracy gain.

\section{Regularized Loss Minimization for Graph Classification}

To support graph classification, state-of-the-art algorithms [23, 10] use a set of subgraphs discovered from the training graphs as features, where each subgraph $g_{k}$ can be used to represent a graph $G_{i}$ as follows:

$$
\hbar_{g_{k}}\left(G_{i}\right)=2 I\left(g_{k} \subseteq G_{i}\right)-1
$$

Here $I(a)=1$ if $a$ holds, and 0 otherwise. This rule simply maps a graph $G_{i}$ into +1 if $g_{k} \subseteq G_{i}$, or -1 otherwise.

Let $\mathcal{F}=\left\{g_{1}, \cdots, g_{m}\right\}$ be the full set of subgraphs for the training graphs. We can use $\mathcal{F}$ as features to represent each graph $G_{i}$ into a vector space as $\boldsymbol{x}_{i}=\left\{\hbar_{g_{1}}\left(G_{i}\right), \cdots, \hbar_{g_{m}}\left(G_{i}\right)\right\}$, with $\boldsymbol{x}_{i}^{k}=\hbar_{g_{k}}\left(G_{i}\right)$. In the following subsection, $G_{i}$ and $\boldsymbol{x}_{i}$ are used interchangeably as they both refer to the same graph. Given the full subgraph features $\mathcal{F}$, the prediction function for the graph $\boldsymbol{x}_{i}$ is a linear classifier:

$$
f\left(\boldsymbol{x}_{i}\right)=\boldsymbol{x}_{\boldsymbol{i}} \cdot \boldsymbol{w}+b=\sum_{g_{k} \in \mathcal{F}} w_{k} \hbar_{g_{k}}\left(G_{i}\right)+b
$$

where $\boldsymbol{w}=\left[w_{1}, \cdots, w_{m}\right]^{\prime}$ is the weight vector for all features $\mathcal{F}$, and $b$ is the bias of the model. The predicted class of $\boldsymbol{x}_{i}$ is +1 if $f\left(\boldsymbol{x}_{i}\right)>0$ or -1 otherwise. Note that in practice, subgraph space $\mathcal{F}$ is implicit and exponentially large, i.e., the number of subgraphs grows exponentially with respect to the number of nodes.

\subsection{Regularized Loss Minimization Formulation}

In this paper, we propose to learn a $K$-dimensional feature space from the implicit and large subgraph space $\mathcal{F}$ to achieve the lowest regularized empirical risks for the 
graph dataset, with $K$ being automatically determined. Eq.(1) can be reformulated as the following objective function:

$$
\min \mathcal{J}(\boldsymbol{w}, b)=\min _{\boldsymbol{w}, b} \underbrace{\frac{1}{n} \sum_{i=1}^{n} \mathcal{L}\left(y_{i}, \boldsymbol{x}_{\boldsymbol{i}} \cdot \boldsymbol{w}+b\right.}_{C})+\underbrace{\gamma_{1}\|\boldsymbol{w}\|_{1}+\gamma_{2}\|\boldsymbol{w}\|_{2}^{2}}_{R}
$$

The first term $C$ measures the loss on the training graphs, where $\mathcal{L}\left(y_{i}, f\left(\boldsymbol{x}_{i}\right)\right)$ can be any loss function measuring the misclassification penalty of a graph $G_{i}$. The second part $R$ consists of regularization terms to enforce sparse and robust solutions. Parameters $\gamma_{1}$ and $\gamma_{2}$ are used to trade-off these parts $\left(\gamma_{1} \geq 0, \gamma_{2} \geq 0\right)$. For the regularization, our objective is to obtain a sparse and stable solution on $\boldsymbol{w}$, i.e., low dimensional subgraph features for final graph classification. Here, we combine both $\ell_{1}$ and $\ell_{2}$ norm, which is known as elastic net in machine learning [21]. The motivation of our regularization is as follows:

The $\ell_{1}$-norm regularizer $\left(\sum_{k}\left|w_{k}\right|\right)$ can produce solutions with many coefficients being 0 , which is known as lasso [20] and has been widely applied for variable selections.

Although $\ell_{1}$ regularization can produce a sparse solution, it suffers from two major disadvantages: (1) the number of selected variables is limited by the number of observations; and (2) the lasso penalized model can only select one variable from a group of correlated variables and does not care which one is selected [21]. In contrast, $\ell_{2}$ regularization, which is widely used in SVM formulation $\left(\left(\|\boldsymbol{w}\|_{2}^{2}=\sum_{k=1}^{m}\left|w_{k}\right|^{2}\right)\right)$, can produce more stable and robust classification results. However, $\ell_{2}$ formulation cannot produce a sparse solution. By combining $\ell_{1}$ and $\ell_{2}$ norm, known as elastic net [21], we can overcome these issues and enjoy the sparse and stable properties.

\subsection{Sparse Subgraph Learning: Challenges and Solution Overview}

Challenges: For explicit vector data with moderate feature size, the problem defined in Eq.(4) can be effectively solved in traditional supervised learning. However, for graph data the challenges are evident: (1) the feature set $\mathcal{F}$ is unavailable (implicit) unless we enumerate all subgraphs from the training graphs, which is NP-complete; and (2) the whole subgraph set is exponentially large, and only a small subset of subgraphs are useful for classifiers to achieve maximum graph classification accuracy. 
Solution Overview: To solve the aforementioned challenges, we propose a regularized loss minimization-driven (RLMD) subgraph selection method for graph classification. Driven by our formulation in Eq.(4), our principle is to iteratively mine the best subgraph feature to reduce the empirical loss on the training graphs. To this end, we resort to the subgradient method in the functional space to define the utility of each subgraph, and embed the feature selection/ranking into the subgraph mining/enumeration process. To handle the exponentially large subgraph space, we derive an effective branch-and-bound pruning scheme to reduce the search space. After a new subgraph is selected, we include and re-solve the new restricted objective function of Eq.(4) by using currently selected subgraphs. To find optimal $K$ value, we derive a stopping criterion for our feature selection procedure based on the subgradient in the functional space, so that $K$ can be automatically obtained once the algorithm converges.

Logistic Loss Function: A Running Example. Our method is based on the gradient/subgradient on the functional space of the objective function Eq.(4). In this paper, we use the logistic loss function as an example to illustrate how subgraph selection is performed by subgradient methods, and the logistic loss function is given as follows:

$$
\mathcal{L}\left(y_{i}, f\left(x_{i}\right)\right)=\log \left(1+\exp \left\{-y_{i} f\left(\boldsymbol{x}_{i}\right)\right\}\right)
$$

Note that our algorithm is a general method in the sense that any other differentiable loss function, such as least square loss $\mathcal{L}\left(y, f_{t}\right)=\frac{1}{2}\left(y-f_{t}\right)^{2}$ or exponential loss $\mathcal{L}\left(y, f_{t}\right)=$ $\exp \left\{-y f_{t}\right\}$, can be directly used in our algorithm. As discussed latter in Section 4.6, our method is also applicable to convex locally Lipschitz but non-differentiable loss functions such as the hinge loss used by the (margin) perceptron and linear SVM.

The partial derivative of the loss term $C$ in Eq.(4) on the subgraph feature $g_{k}$ is defined as $\frac{\partial C}{\partial w_{k}}$. For logistic loss function,

$$
\begin{aligned}
\frac{\partial \mathcal{C}}{\partial w_{k}} & =\frac{1}{n} \sum_{i=1}^{n} \frac{\partial \mathcal{L}\left(y_{i}, f\left(\boldsymbol{x}_{i}\right)\right)}{\partial f\left(\boldsymbol{x}_{i}\right)} \frac{\partial f\left(\boldsymbol{x}_{i}\right)}{\partial w_{k}} \\
& =-\frac{1}{n} \sum_{i=1}^{n} \frac{y_{i} \boldsymbol{x}_{i}^{k}}{1+e^{i} i_{i}\left(\boldsymbol{x}_{i}\right)}=\sum_{i=1}^{n} y_{i} \alpha_{i} \boldsymbol{x}_{i}^{k}
\end{aligned}
$$

Here, $\left.\alpha_{i}=-\frac{1}{n\left(1+e^{y} i f\left(x_{i}\right)\right.}\right)$ can be regarded as a weight associated with graph $G_{i}$ for the subgraph mining process. 


\subsection{RLMD Subgraph Selection for Graph Classification}

Because we aim to learn a sparse solution of subgraph features ( $K$-dimensional feature space) from graph data, some subgraphs/features $g_{k}$ with zero weights, i.e., $w_{k}=0$ will not be used for learning the classification model. Thus it makes sense to partition the subgraph features $\mathcal{F}$ into two disjoint subsets $\mathcal{F}_{1}$ and $\mathcal{F}_{2}$. $\mathcal{F}_{1}$ stores active features which are used to learn the classification model and this set is frequently updated as desired, and $\mathcal{F}_{2}$ includes unselected graphs with 0 weights (i.e., for $g_{k} \in$ $\mathcal{F}_{2}, w_{k}=0$ ). Then we can iteratively select the best feature from $\mathcal{F}_{2}$ to $\mathcal{F}_{1}$, and solve the following restricted subproblem:

$$
\begin{aligned}
\min \mathcal{J}_{t}(\boldsymbol{w}, b) & =\min _{\boldsymbol{w}, b} \underbrace{\frac{1}{n} \sum_{i=1}^{n} \mathcal{L}\left(y_{i}, \boldsymbol{x}_{i}(t) \cdot \boldsymbol{w}+b\right)}_{C^{\prime}}+\underbrace{\gamma_{1}\|\boldsymbol{w}\|_{1}+\gamma_{2}\|\boldsymbol{w}\|_{2}^{2}}_{R^{\prime}} \\
& =\mathcal{J}_{t}(\boldsymbol{w}(t), b(t))
\end{aligned}
$$

where $\boldsymbol{x}_{i}(t)$ is the feature representations for graph $G_{i}$ based on the active set $\mathcal{F}_{1}$ in the $t$-th iteration, and $(\boldsymbol{w}(t), b(t))$ is the solution of Eq.(7). Note that $\mathcal{J}_{t}(\boldsymbol{w}, b)$ is used here to denote the restricted subproblem Eq.(7) while $\mathcal{J}(\boldsymbol{w}, b)$ is referred to original problem in Eq.(4).

The optimal number of subgraphs $K$ can be automatically determined by setting $K=\left|\mathcal{F}_{1}\right|$ once the algorithm converges. Note that given a solution $(\boldsymbol{w}(t), b(t))$, the loss term $C^{\prime}$ in Eq.(7) equals to $C$ in Eq.(4) because the prediction of of graph mainly depends on the active set $\mathcal{F}_{1}$, i.e., $C^{\prime}=C$. In the following, we will derive the stopping condition of our algorithm, and prove its convergence.

Stopping Condition for Optimal $K$ value: Our objective function Eq.(4) is convex and non-smooth, i.e., it may be non-differentiable at a point $w$. When it is nondifferentiable at $\boldsymbol{w}$, we can compute its generalized gradient (i.e., subgradient) instead. According to the optimization conditions, when reaching the optimum, we will have

$$
0 \in \frac{\partial C}{\partial w_{k}}+\gamma_{1} o_{k}+2 \gamma_{2} w_{k}
$$

where $o_{k}$ is the subgradient with respect to $w_{k}$

$$
o_{k} \in\left\{\begin{array}{rll}
\operatorname{sign}\left(w_{k}\right) & : & w_{k} \neq 0 \\
{[-1,1]} & : & w_{k}=0
\end{array}\right.
$$


where $\operatorname{sign}(a)=1$ if $a>0$ otherwise -1 .

According to Eq.(8) and Eq.(9), we can now state the optimal condition for our sparse subgraph learning problem.

Proposition 1. Optimal Solution: Let $\hat{\boldsymbol{w}}=\left[\hat{w}_{1}, \cdots, \hat{w}_{m}\right]$. Suppose that $(\hat{\boldsymbol{w}}, \hat{b})$ is the optimal solution of our objective function Eq.(4), then Eq.(10) and Eq.(11) hold.

$$
\begin{array}{rrr}
\frac{\partial C}{\partial \hat{w}_{k}}+\gamma_{1} \operatorname{sign}\left(\hat{w}_{k}\right)+2 \gamma_{2} \hat{w}_{k} & =0 \text { if } \quad \hat{w}_{k} \neq 0 \\
\left|\frac{\partial C}{\partial \hat{w}_{k}}\right| \leq \gamma_{1} & \text { if } \quad \hat{w}_{k}=0
\end{array}
$$

Eq.(11) holds because for $\hat{w}_{k}=0$, the third term of Eq.(8) disappears. Combining Eq.(8) and Eq.(9) will result in Eq.(11).

To reduce the objective value $\mathcal{J}_{t}$ in Eq.(7), we propose to select a subgraph in $\mathcal{F}_{2}$ whose weight violates Eq.(11), and update the selected active set $\mathcal{F}_{1}$ with the newly selected feature and re-optimize the restricted subproblem Eq.(7) with current features. This process will repeat until no candidate violates Eq.(11). In other words, Eq.(11) is a stopping condition and determines the number of subgraphs being selected for RLMD's subgraph selection process.

Utility of Subgraphs: Eq.(11) can be used naturally to induce a criterion for quantifying the utility value of a subgraph. The larger $\left|\frac{\partial C}{\partial w_{k}}\right|$ is, the more informative it will be for reducing the objective function. Accordingly, we formally define the informative score as follows:

Definition 3. Informative Score: For a subgraph pattern $g_{k}$, its informative score for graph classification is defined as follows:

$$
\Theta\left(g_{k}\right)=\left|\frac{\partial C}{\partial w_{k}}\right|=\left|\frac{\partial C^{\prime}}{\partial w_{k}}\right|=\left|\sum_{i=1}^{n} y_{i} \alpha_{i} x_{i}^{k}\right|
$$

where $\alpha_{i}=-\frac{1}{n\left(1+e^{y_{i} f\left(x_{i}\right)}\right)}$.

Note that the informative score directly depends on the weight of each graph $\alpha_{i}$, which is calculated based on the active set $\mathcal{F}_{1}$. Intuitively, the best subgraph of $\mathcal{F}_{2}$ is the one with the maximum informative score, because it is more likely to violate the stopping condition Eq.(11). 


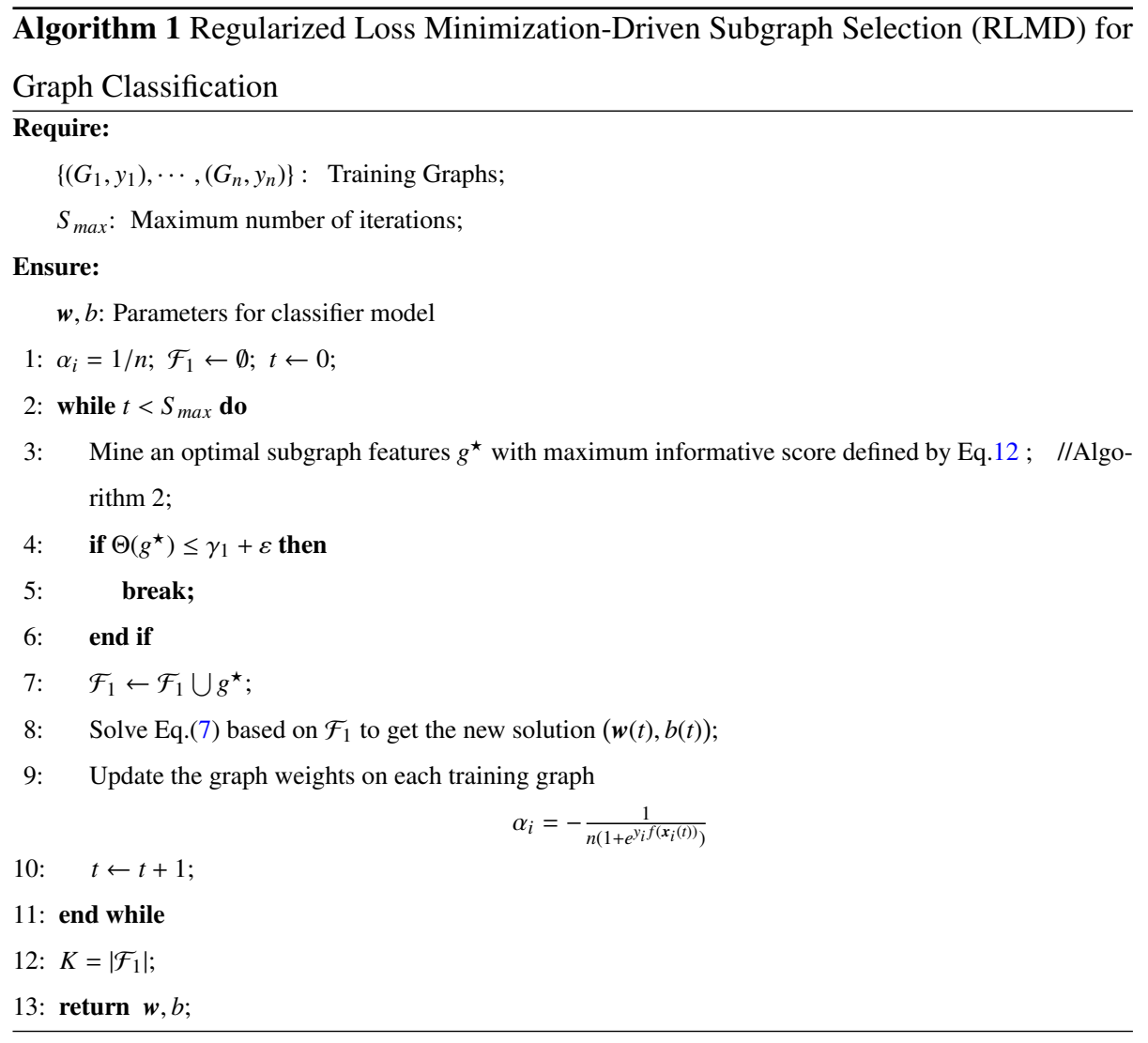

RLMD Algorithm: Algorithm 1 illustrates the detailed steps of RLMD for graph classification. Initially, the weights for all training graphs are equally set as $1 / n$, and the active set $\mathcal{F}_{1}$ is initialized to be empty.

In the next step, the algorithm mines an optimal subgraph $g^{\star}$ from $\mathcal{F}_{2}$ which has the highest informative scores defined by Eq.(12). This step involves a subgraph mining procedure, which will be addressed in the next subsection. On steps 4-5, if current optimal subgraph no longer violates the optimal condition Eq.(11), the algorithm terminates. Here, we have relaxed the convergence condition to $\varepsilon$ tolerance; this is because in the last few iterations, the maximum score will only change subtly (we set $\epsilon=0.005$ in our experiments).

On step 7, we add the newly selected subgraph $g^{\star}$ to the existing subgraph set $\mathcal{F}_{1}$, and re-solve the following restricted subproblem Eq.(7). To solve this restricted 
objective function, we use the MALSAR toolbox ${ }^{2}$ in our experiments. It is worth noting that because this step only involves a very small number of features, it is very efficient in practice.

Subsequently, the algorithm updates the weight $\alpha_{i}$ for each graph $G_{i}$. This will help compute the derivative of $\frac{\partial C}{\partial w_{k}}$ for subgraph mining in next round. After the algorithm terminates, the optimal number of subgraphs $K$ can be easily obtained as $K=\left|\mathcal{F}_{1}\right|=t$ on step 12 .

Note that our algorithm 1 generalizes the column generation technique in gBoost [23] by iteratively selecting the most violated subgraph in each iteration until convergence. Our algorithm 1 relies on $\varepsilon$ and $\gamma_{1}$, which serve as a stopping condition to determine $K$. In practice, $\varepsilon$ is a subtle value insensitive to the algorithm performance. Meanwhile, $\gamma_{1}$ is much easier to set than asking users to specify $K$ values because $\gamma_{1}$ is chosen in a much smaller range, as we will demonstrate in Section 5.2.4.

\subsection{Theoretical Study}

Theorem 1. (Convergence Property:) Algorithm 1 guarantees that the restricted objective function Eq.(7) will monotonically decrease.

Proof. Suppose in the t-th iteration, the optimal objective value based on current $t$ features (i.e., $\left.\left|\mathcal{F}_{1}\right|=t\right)$ is obtained with $(\boldsymbol{w}(t), b(t))$, i.e.,

$\mathcal{J}_{t}(\boldsymbol{w}(t), b(t))=\underbrace{\frac{1}{n} \sum_{i=1}^{n} \mathcal{L}\left(y_{i}, \boldsymbol{x}_{i}(t) \cdot \boldsymbol{w}(t)+b(t)\right)}_{C=C^{\prime}}+\underbrace{\gamma_{1}\|\boldsymbol{w}(t)\|_{1}+\gamma_{2}\|\boldsymbol{w}(t)\|_{2}^{2}}_{\mathcal{R}=\mathcal{R}^{\prime}}=(C+\mathcal{R})_{\mid(w(t), b(t))}$ then in the $t+1$-th iterations, the optimal objective value for Eq.(7) is

$$
\min \mathcal{J}_{t+1}(\boldsymbol{w}, b)=\min (C+\mathcal{R}) \leq(C+\mathcal{R})_{\mid([w(t), 0], b(t))}=\mathcal{J}_{t}(\boldsymbol{w}(t), b(t))
$$

Here $[w(t), 0]$ means that the weights for subgraphs selected in the $t$-th iteration remain unchanged while the weight for newly selected subgraph in the $t+1$-th iteration is 0 .

\footnotetext{
${ }^{2}$ http://www.MALSAR.org
} 
Thus the objective value of the restricted problem Eq.(7) based on the currently selected features $\mathcal{F}_{1}$ always monotonously decreases in two successive iterations. Because the objective function value is non-negative (bounded), we can ensure that it will finally converge as iteration continues. The proof is complete.

Suppose the algorithm converges in the $K$-th iteration with a solution $(\boldsymbol{w}(K), b(K))$, and the objective value for Eq.(7) is $\mathcal{J}_{K}(\boldsymbol{w}(K), b(K))$. By adding $m-K$ zeros for subgraphs in $\mathcal{F}_{2}$ to $\boldsymbol{w}(K), i, e$, $\hat{\boldsymbol{w}}(K)=[\boldsymbol{w}(K), 0 \cdots]$, we obtain a solution $(\hat{\boldsymbol{w}}(K), b(K))$ for Eq.(4).

Corollary 1. Optimal Solution Guarantees: If Algorithm 1 converges with solution $(\boldsymbol{w}(K), b(K))$ for Eq.(7), then $(\hat{\boldsymbol{w}}(K), b(K))$ is an optimal solution for Eq.(4).

ProOF. According to our Proposition 1, $(\hat{\boldsymbol{w}}(K), b(K))$ is an optimal solution of Eq.(4), because $\forall w_{k}=0\left(g_{k} \in \mathcal{F}_{2}\right)$, we have $\Theta\left(g_{k}\right)<\gamma_{1}$ based on our stopping condition. Thus we will have

$$
\mathcal{J}_{K}(\boldsymbol{w}(K), b(K))=\mathcal{J}(\hat{\boldsymbol{w}}(K), b(K))=\min \mathcal{J}(\boldsymbol{w}, b)
$$

where $J_{K}(\boldsymbol{w}(K), b(K))$ and $\mathcal{J}(\hat{\boldsymbol{w}}(K), b(K))$ refer to the objective values of the restricted subproblem Eq.(7) and Eq.(4), respectively.

We have proved that objective value for Eq.(7) is monotonously decreasing (Theorem 1) and its recovered solution $(\hat{\boldsymbol{w}}(K), b(K))$ is an optimal solution to Eq.(4) (Corollary 1).

\subsection{Optimal Subgraph Mining}

In order to mine optimal subgraph $g^{\star}$ on step 3 of Algorithm 1, we need to perform the subgraph enumeration procedure. In RLMD, we employ the frequent subgraph mining-based algorithm gSpan [42]. The key idea of gSpan is that each subgraph has a unique DFS Code, which is defined by a lexicographic order of the discovery time during the search process. By employing a depth first search strategy on the DFS Code tree (where each node is a subgraph), gSpan can enumerate all frequent subgraphs efficiently. 
385

During the subgraph mining process, the search space is exponentially large, which requires an effective pruning scheme to reduce the search space. In this subsection, we will derive the upper-bound of the informative score for each subgraph, which helps prune the search space and speed up the subgraph mining.

Theorem 2. (Upper-bound Score:) Let $g$ and $g^{\prime}$ be two subgraph patterns, and $g \subseteq g^{\prime}$,

for the subgraph $g$, we define

$$
\begin{aligned}
A_{1}(g) & =2 \sum_{\left\{i \mid y_{i}=+1, g \in G_{i}\right\}} \alpha_{i} \\
A_{2}(g) & =2 \sum_{\left\{i \mid y_{i}=-1, g \in G_{i}\right\}} \alpha_{i} \\
A_{3} & =\sum_{t=1}^{n} \alpha_{i} y_{i}
\end{aligned}
$$

$$
\hat{\Theta}(g)=\left\{\begin{array}{lll}
\max \left\{\left|A_{1}(g)-A_{3}\right|,\left|A_{2}(g)\right|\right\} & : & A_{3} \geq 0 \\
\max \left\{\left|A_{2}(g)+A_{3}\right|,\left|A_{1}(g)\right|\right\} & : & A_{3}<0
\end{array}\right.
$$

then $\Theta\left(g^{\prime}\right) \leq \hat{\Theta}(g)$, where $\Theta\left(g^{\prime}\right)$ is defined in Eq.(12).

Proof. We start with the definition of $\Theta\left(g^{\prime}\right)$ :

$$
\begin{aligned}
& \Theta\left(g^{\prime}\right)=\left|\sum_{i=1}^{n} y_{i} \alpha_{i} \boldsymbol{x}_{i}^{\prime}\right| \\
& =\left|\sum_{i=1}^{n} y_{i} \alpha_{i} \cdot\left[2 I\left(g^{\prime} \subseteq G_{i}\right)-1\right]\right| \\
& =\left|2 \sum_{g^{\prime} \subseteq G_{i}} y_{i} \alpha_{i}-\sum_{i=1}^{n} \alpha_{i} y_{i}\right| \\
& =\left|A_{1}\left(g^{\prime}\right)-A_{2}\left(g^{\prime}\right)-A_{3}\right| \\
& \leq\left\{\begin{array}{lll}
\max \left\{\left|A_{1}\left(g^{\prime}\right)-A_{3}\right|,\left|A_{2}\left(g^{\prime}\right)\right|\right\} & : & A_{3} \geq 0 \\
\max \left\{\left|A_{2}\left(g^{\prime}\right)+A_{3}\right|,\left|A_{1}\left(g^{\prime}\right)\right|\right\} & : & A_{3}<0
\end{array}\right. \\
& \leq\left\{\begin{array}{lll}
\max \left\{\left|A_{1}(g)-A_{3}\right|,\left|A_{2}(g)\right|\right\} & : & A_{3} \geq 0 \\
\max \left\{\left|A_{2}(g)+A_{3}\right|,\left|A_{1}(g)\right|\right\} & : & A_{3}<0
\end{array}\right. \\
& =\hat{\Theta}(g)
\end{aligned}
$$

.

$$
\text { bou }
$$

up

91

The first inequality holds because for $\alpha_{i}<0, A_{1}\left(g^{\prime}\right) \leq 0$ and $A_{2}\left(g^{\prime}\right) \leq 0$, so the upperbound depends on $A_{3}$. If $A_{3} \geq 0, A_{1}\left(g^{\prime}\right)$ and $A_{3}$ will have different signs, then the upper-bound is the maximum between $\left\{\left|A_{1}\left(g^{\prime}\right)-A_{3}\right|,\left|A_{2}\left(g^{\prime}\right)\right|\right\}$. The case is similar for $A_{3}<0$. The second inequality holds because $\left|A_{1}\left(g^{\prime}\right)\right| \leq\left|A_{1}(g)\right|$ and $\left|A_{2}\left(g^{\prime}\right)\right| \leq\left|A_{2}(g)\right|$ for $g \subseteq g^{\prime}$. 


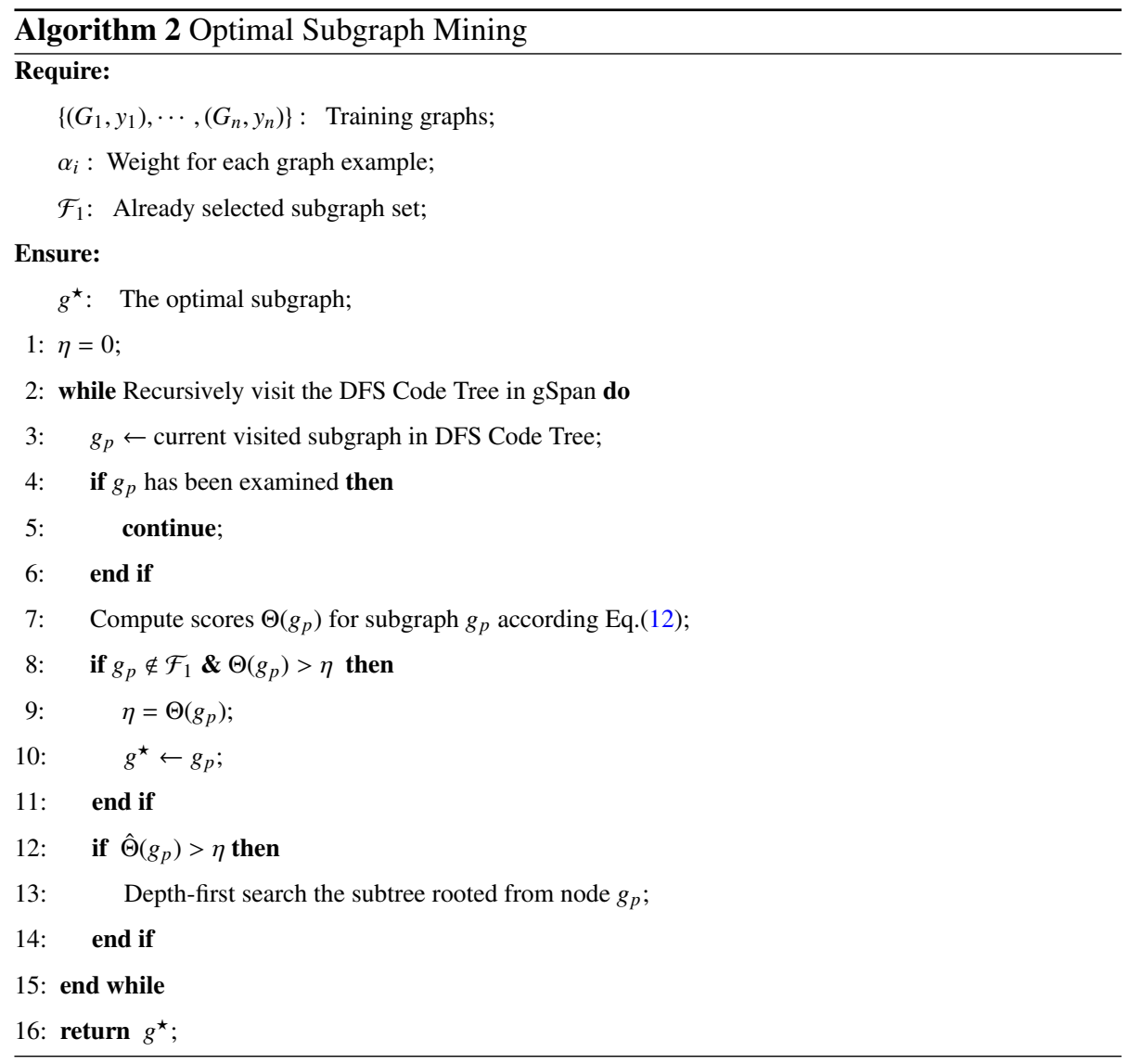

Theorem 2 states that for any super graph of a subgraph $g$, its informative score is upper-bounded by $\hat{\Theta}(g)$. This rule can prune unpromising candidates effectively.

Optimal Subgraph Exploration Algorithm: Our optimal subgraph mining algorithm is listed in Algorithm 2. The minimum value $\eta$ in the optimal set is initialized on step 1. Duplicated subgraph features are pruned on steps 4-5, and the informative score $\Theta\left(g_{p}\right)$ for $g_{p}$ is calculated on step 7. If $g_{p}$ is not selected before $\left(g_{p} \notin \mathcal{F}_{1}\right)$ and $\Theta\left(g_{p}\right)$ is larger than $\eta$, we replace the optimal subgraph $g^{\star}$ with the current $g_{p}$ and update the optimal score $\eta$ (steps 8-11).

A branch-and-bound pruning rule, according to Theorem 2 , is subsequently used to prune the search space on step 12. Lastly, the optimal subgraph $g^{\star}$ is returned on step 16. 
The above pruning process is a key feature of our algorithm, because we do not require a support threshold value for subgraph mining (whereas all filter subgraph mining methods will require users to predefine a threshold value in order to discover subgraphs).

\subsection{Relation to gBoost}

Our RLMD subgraph selection algorithm advances the existing column generation style techniques employed in gBoost [23] for graph classification. The learning objective function for $\mathrm{gBoost}$ is

$$
\begin{aligned}
\max _{\rho, \boldsymbol{w}, \boldsymbol{\xi}} & \rho-\frac{1}{v n} \sum_{i=1}^{n} \xi_{i} \\
\text { s.t. } & y_{i} \sum_{k=1}^{m} \hbar_{g_{k}}\left(G_{i}\right) w_{k}+\xi_{i} \geq \rho ; \\
& \sum_{k=1}^{m} w_{k}=1 ; \\
& w_{k} \geq 0, \xi_{i} \geq 0 ;
\end{aligned}
$$

From [43], we know that this formula is equivalent to the following linear programming:

$$
\begin{array}{cl}
\min _{w, \xi} & \sum_{k=1}^{m} w_{k}+C \sum_{i=1}^{n} \xi_{i} \\
\text { s.t. } & y_{i} \sum_{k=1}^{m} \hbar_{g_{k}}\left(G_{i}\right) w_{k}+\xi_{i} \geq 1 ; \\
& w_{k} \geq 0, \xi_{i} \geq 0 ;
\end{array}
$$

Eq.(14) is actually a $\ell_{1}$ svm formulation, and can also be formulated as the regularized loss minimization formulation problem:

$$
\min \|\boldsymbol{w}\|_{1}+C \sum_{i=1}^{n} \mathcal{L}_{h}\left(y_{i}, f\left(\boldsymbol{x}_{i}\right)\right)
$$

Here, $\mathcal{L}_{h}\left(y_{i}, f\left(\boldsymbol{x}_{i}\right)\right)=\max \left(1-y_{i} f\left(\boldsymbol{x}_{i}\right), 0\right)$, which is known as hinge loss in machine learning.

Compared to our objective function in Eq.(4), we can find that gBoost (Eq.15) is a special case of Eq.(4), with the $\ell_{2}$ regularization term being 0. Although the hinge loss function is non-differentiable, our subgradient method still applies, as long as $\frac{\partial C}{\partial w_{k}}$ in Eq.(6) is properly defined. This observation shows the following advantages of our algorithm: (1) gBoost employs a hinge loss function which is similar to SVM and 
requires the problem to be formulated as a linear programming. Our algorithm generalizes and advances gBoost by removing the linear programming constraint and can employ any differentiable loss function, in addition to the logistic loss function considered in our paper. This generalization has great attractiveness in many applications, especially when the probability estimation for classification is required (the logistic function can provide some probabilistic information compared to the hinge loss function); (2) while gBoost employs $\ell_{1}$ norm regularization to obtain a sparse solution, our algorithm considers an additional norm $\ell_{2}$. This combined norm (known as elastic net) enables a sparse and more stable solution.

\section{Experiment}

\subsection{Experimental Settings}

Benchmark Data: We validate the performance of the proposed algorithm on two types of graph classification datasets.

Anti-cancer activity prediction (NCI): The NCI graph collection ${ }^{3}$ is a benchmark for predicting the biological activity of small molecules for different types of cancers. Each NCI dataset belongs to a bioassay task for anticancer activity prediction, such as Breast cancer or Leukemia cancer. Each molecule is represented as a graph, with atoms representing nodes and bonds denoting edges. A molecule is positive if it is active against a certain type of cancer, or negative otherwise. Table 1 summarizes nine NCI graph classification tasks used in our experiments, where columns 2-4 denote the number of positive molecules, the total number of graphs, and the type of cancer of each dataset. In our experiments, we randomly select 1000 graphs from each dataset with balanced class distributions for graph classification.

Predictive Toxicology Challenge Dataset (PTC): The PTC challenge includes a number of carcinogenicity classifications for the toxicology prediction of chemical compounds ${ }^{4}$. The dataset we selected contains 417 compounds with four types of test animals: MM (male mouse), FM (female mouse), MR (male rat), and FR (female

\footnotetext{
${ }^{3}$ http://pubchem.ncbi.nlm.nih.gov

${ }^{4} \mathrm{http}: / /$ www.predictive-toxicology.org/ptc/
} 
Table 1: Datasets Used in Experiments

\begin{tabular}{cccc}
\hline ID & \#Pos & \#Total & Learning tasks \\
\hline 1 & 1793 & 37349 & Non-Small Cell Lung \\
33 & 1467 & 37022 & Melanoma \\
41 & 1350 & 25336 & Prostate \\
47 & 1735 & 37298 & Central Nerv Sys \\
81 & 2081 & 37549 & Colon \\
83 & 1959 & 25550 & Breast \\
109 & 1773 & 37518 & Ovarian \\
123 & 2715 & 36903 & Leukemia \\
145 & 1641 & 37043 & Renal \\
\hline
\end{tabular}

rat). Each compound has labels selected from $\{\mathrm{CE}, \mathrm{SE}, \mathrm{P}, \mathrm{E}, \mathrm{EE}, \mathrm{IS}, \mathrm{NE}, \mathrm{N}\}$. Similar to [44], we set $\{\mathrm{CE}, \mathrm{SE}, \mathrm{P}\}$ as positive labels, and $\{\mathrm{NE}, \mathrm{N}\}$ as negative labels.

Baseline Methods: In our experiments, we consider three types of baseline methods, namely the two-step filter methods (TFMs), direct filter methods (DFMs), and embedded methods, as follows:

- IG+SVM is a TFM method that simply mines a set of frequent subgraphs, and then performs feature selection by using Information Gain. A SVM classifier is trained by using selected subgraph features for graph classification.

- TOP+SVM is similar to IG+SVM except that it selects the top $K$ subgraphs based on their frequency rather than their information gain values.

- gSemi+SVM [11] is a DFM method, which integrates the feature selection into the subgraph mining process. The measurement for feature selection mainly considers the must-link and cannot link constraints between graph samples within the same or between different classes.

- gHSIC+SVM [12] is another DFM method which exploits the correlations between features and labels.

- gBoost [23] is a state-of-the-art embedded method which formulates the feature selection as a linear problem and selects subgraph features which best fit the 
objective function.

- RLMD is our proposed method which employ a logistic loss function together with an elastic net for regularization, and automatically determines optimal number of subgraphs $K$.

We conduct 10-fold cross-validation on all graph datasets and report the average results and standard errors of 10 folds in the final result. The parameters for $\gamma_{1}$ are selected from $\{0.005,0.01,0.03,0.05\}$, and $\gamma_{2}$ is selected from $\{0.01,0.03,0.05\}$. We will further analyze the impact of $\gamma_{1}$ and $\gamma_{2}$ in wider ranges in Section 5.2.4. For the filter methods (IG+SVM, TOP+SVM, gSemi+SVM, and gHSIC+SVM), the minimum support for frequent subgraph mining is set to $10 \%$ on NCI graph datasets and $1 \%$ on PTC classification tasks, and an SVM classifier is trained with $C$ parameter from the range $\{0.1,1,10,100,1000,10000\}$. For the gBoost algorithm, the parameter $v$ is selected from $\{0.1,0.2,0.3,0.4\}$. Following [23], we select the best average results of 10 -fold cross-validation for each baseline algorithm by varying these parameters, which represents the best performance each baseline can achieve.

For fairness of comparison, we increase the number of features to be selected for the filter methods (IG+SVM, TOP+SVM, gSemi+SVM, and gHSIC+SVM), and increase the iterations for the embedded methods (gBoost and RLMD), then collect and compare the performance of all algorithms under the same number of features. We set $S_{\max }=200$, which defines the maximum number of features used to learn the classifier models. Note that for RLMD, the algorithm may stop before reaching the maximum iterations/subgraphs we set, i.e., the optimal $K$ is obtained. When RLMD stops, the optimal number of subgraph features has been discovered and RMLD will not add additional subgraphs to the feature set. We also compare RLMD under the optimal subgraph value to other baselines with the same number of $K$ features (the purpose is to show that the optimal subgraph features discovered by RLMD are indeed optimal for graph classification). 


\subsection{Experimental Results}

\subsubsection{Results on NCI Graph Dataset}

For the NCI graph datasets, we vary the number of selected subgraph features from 20 to 200 for filter methods, and the number of iterations for gBoost and RLMD from 1 to 200. The accuracies and AUC values are shown in Fig. 3.

Comparison with Filter methods: The results in Fig. 3 show that with the increase in the number of features/iterations, the filter methods (TOP+SVM, IG+SVM, gSemi+SVM, and gHSIC+SVM) are inferior to RLMD. This is because filter methods separate the feature selection module from the model learning process. The subgraph features selected from filter methods may not fit the underlying learning model very well (we use SVM in our experiments). This is actually an observed common drawback of filter methods [19]. The performance among these filter-based methods varies from one graph dataset to another, and none of them significantly outperforms others. For instance, gSemi+SVM outperforms TOP+SVM, IG+SVM, and gHSIC+SVM on NCI-1 (Fig. 3.A) when the number of selected graphs is considerably large ( $\geq 160)$, but is worse than gHSIC on NCI-109 (Fig. 3.G). This may be attributed to the inherent differences underneath the graph datasets.

How many subgraphs to select: Another drawback of filter methods, shown in our experiments, is that the performance of filter methods varies significantly w.r.t. different numbers of selected features $(K)$. Indeed, all these filter methods only select subgraph features with maximum discriminative score regardless of the redundancy among the features. Adding redundant features may decrease the performance of an algorithm. Further analysis of subgraph features is presented with a case study in the next subsection.

In contrast, for embedded methods, the above drawbacks can be handled effectively. Our algorithm RLMD unifies the feature selection and model learning into a whole framework, so that the feature selection process is driven by the well-defined objective function, and the selected features can further enhance the learning models. At the same time, RLMD is guaranteed to be convergent given an appropriate $\gamma_{1}$ value, which means that we do not need to specify the total number of selected graphs $K$. For 

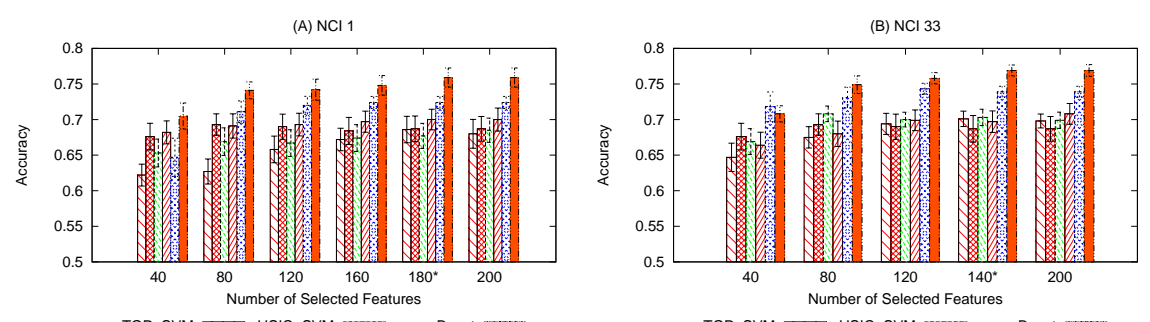

$\underset{\mathrm{IG}+\mathrm{SVM}}{\mathrm{TOP}+\mathrm{SVMIC}+\mathrm{SVM}} \mathrm{gSem+ \textrm {SVM }} \quad \mathrm{gBoost}$

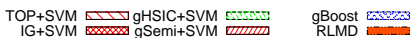
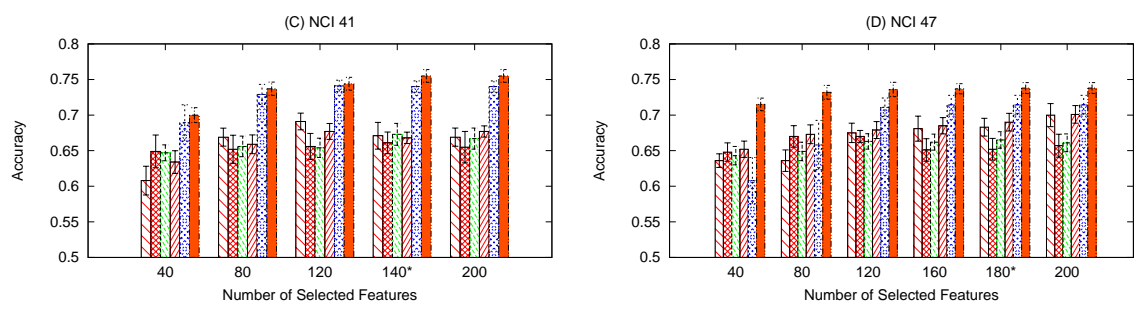

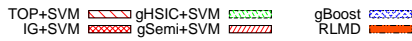

TOP+SVM
IG+SVM gHSIC+SVM
gSemi+SVM
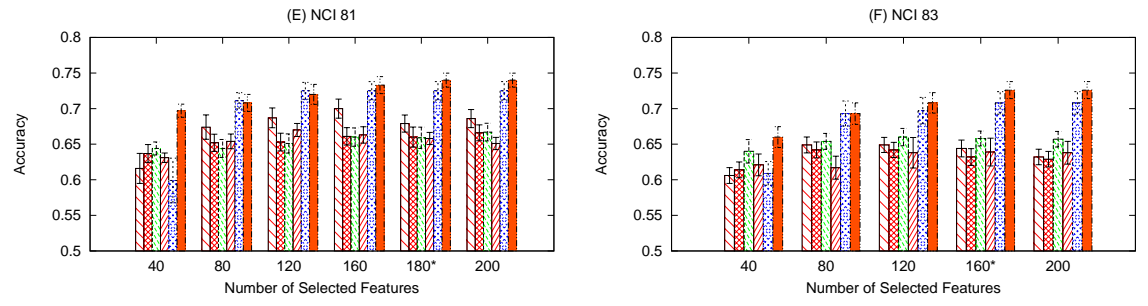

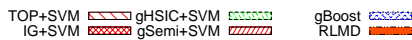

TOP+SVM
IG+SVM
gSemi+SVM
RLMD
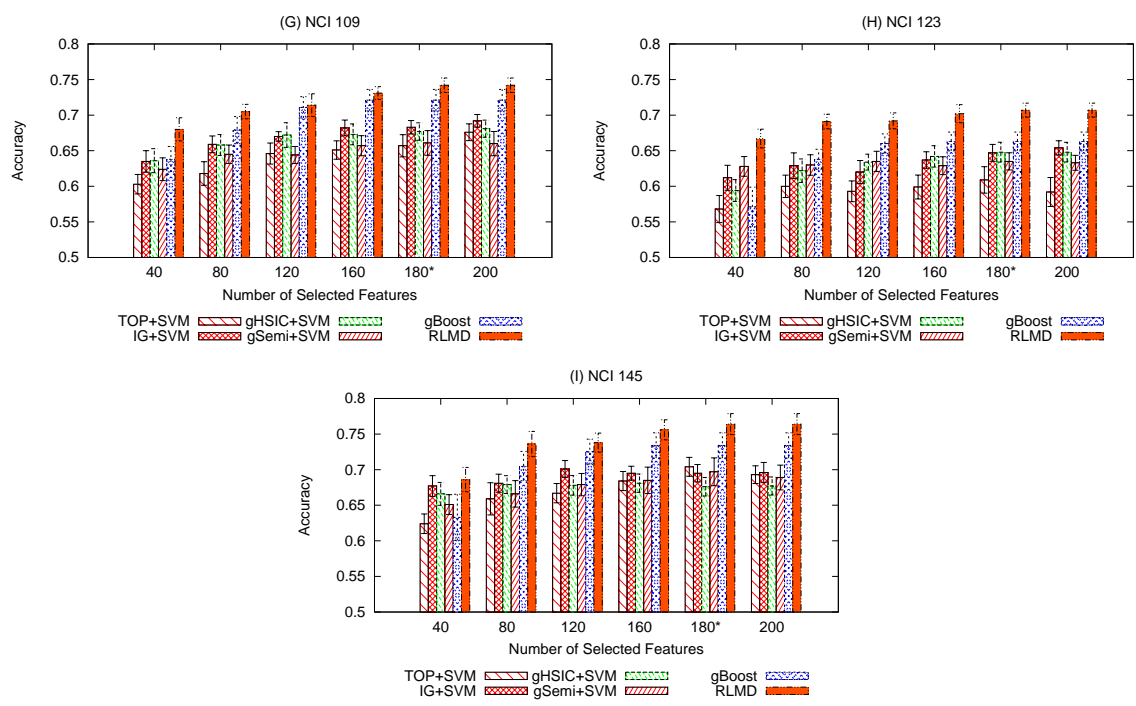

Figure 3: The classification accuracy and standard error on NCI dataset w.r.t. the number of selected graphs. The optimal number of subgraphs selected by RLMD (once it converges for all 10 folds experiments) is marked by a star $*$ at $x$-axis. 
instance, in Fig. (3).A, RLMD reaches convergence with 180 features.

RLMD vs. gBoost: It is evident that RLMD outperforms gBoost for most datasets. This is mainly because gBoost only uses $\ell_{1}$-norm regularization to produce a sparse solution. As pointed out in [21], the lasso $\left(\ell_{1}\right.$-norm) has several drawbacks: (1) when the number of features ( $m$, which is exponentially huge) is much bigger than the number of observations $(n)$, the $\ell_{1}$ norm selects at most $n$ variables before it saturates; and (2) when the pairwise correlations in a group of variables are very high, lasso tends to select only one variable from the group and does not discriminate which one it selects. By contrast, RLMD uses an elastic net (combination of $\ell_{1}$ and $\ell_{2}$ norm), which encourages a grouping effect, where strongly correlated features will be included/excluded. As a result, RLMD results in a similar sparsity of representation to gBoost, but often outperforms gBoost.

Table 2: Averaged accuracies and standard errors on NCI Graphs with Optimal $K$.

\begin{tabular}{|c|c|c|c|c|c|c|}
\hline ID & RLMD & $\begin{array}{c}\text { TOP } \\
+ \text { SVM }\end{array}$ & $\begin{array}{c}\mathrm{IG} \\
+\mathrm{SVM}\end{array}$ & $\begin{array}{l}\text { gHSIC } \\
+ \text { SVM }\end{array}$ & $\begin{array}{l}\text { gSemi } \\
+ \text { SVM }\end{array}$ & gBoost \\
\hline 1 & $\mathbf{0 . 7 5 9} \pm 0.014$ & $0.686 \pm 0.019$ & $0.687 \pm 0.018$ & $0.677 \pm 0.018$ & $0.700 \pm 0.015$ & $0.724_{ \pm 0.009}$ \\
\hline 33 & $\mathbf{0 . 7 6 9} \pm 0.008$ & $0.701 \pm 0.011$ & $0.687 \pm 0.019$ & $0.703 \pm 0.012$ & $0.697 \pm 0.015$ & $0.739 \pm 0.008$ \\
\hline 41 & $\mathbf{0 . 7 5 5} \pm 0.009$ & $0.677 \pm 0.017$ & $0.649 \pm 0.015$ & $0.672 \pm 0.015$ & $0.670 \pm 0.009$ & $0.740 \pm 0.008$ \\
\hline 47 & $\mathbf{0 . 7 3 8}_{ \pm 0.009}$ & $0.683 \pm 0.013$ & $0.652 \pm 0.015$ & $0.665 \pm 0.012$ & $0.690 \pm 0.012$ & $0.714 \pm 0.014$ \\
\hline 81 & $\mathbf{0 . 7 4 0} \pm 0.010$ & $0.679_{ \pm 0.012}$ & $0.660_{ \pm 0.014}$ & $0.659_{ \pm 0.015}$ & $0.658 \pm 0.009$ & $0.725 \pm 0.013$ \\
\hline 83 & $\mathbf{0 . 7 2 6} \pm 0.012$ & $0.644 \pm 0.012$ & $0.632 \pm 0.012$ & $0.658 \pm 0.011$ & $0.639 \pm 0.020$ & $0.708 \pm 0.016$ \\
\hline 109 & $\mathbf{0 . 7 4 2} \pm 0.010$ & $0.657 \pm 0.016$ & $0.683 \pm 0.009$ & $0.677 \pm 0.012$ & $0.661 \pm 0.017$ & $0.721 \pm 0.015$ \\
\hline 123 & $\mathbf{0 . 7 0 7} \pm 0.010$ & $0.609_{ \pm 0.019}$ & $0.647 \pm 0.012$ & $0.648 \pm 0.014$ & $0.635 \pm 0.012$ & $0.663 \pm 0.013$ \\
\hline 145 & $\mathbf{0 . 7 6 4} \pm 0.015$ & $0.704 \pm 0.013$ & $0.695 \pm 0.012$ & $0.676 \pm 0.013$ & $0.697 \pm 0.020$ & $0.734 \pm 0.018$ \\
\hline
\end{tabular}

Overall Performance with Optimal $K$ : In Table 2, we summarize the performance of our algorithm under optimal $K$ value with other methods, where filter methods use the same number of subgraphs $(K)$ for graph classification, and gBoost runs until convergence. The result in Table 2 clearly demonstrates that RLMD outperforms twostep filter methods (TOP+SVM and IG+SVM), direct filter methods (gHSIC+SVM and gSemi+SVM), and gBoost algorithm in NCI datasets. 


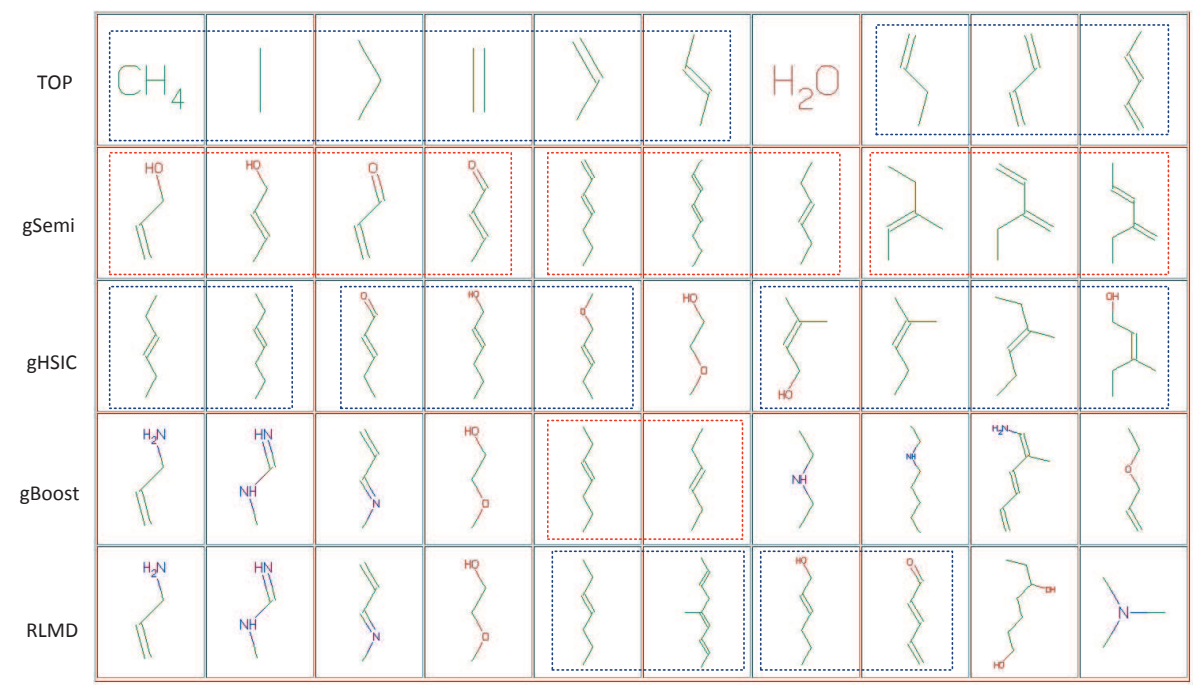

Figure 4: Case Study: comparison of discriminative subgraph features discovered by different algorithms. Subgraph features with high similarities are grouped and highlighted in the dashed rectangles. Subgraphs mined by filter methods are similar to each other and share high redundancy.

\subsubsection{Case Study: Subgraph Feature Comparison}

In this subsection, we use NCI-1 dataset as a case study to investigate subgraphs discovered by different algorithms. In our experiments, the top-10 subgraph features are discovered and illustrated in Fig. 4.

It is evident that the features for all filter methods (TOP, gSemi, and gHSIC) share high correlations. For the gSemi algorithm, for instance, the top-10 subgraphs form 3 groups. In each group, the subgraph features are very similar to each other. This is because the subgraph mining algorithm follows the depth-first-search (DFS) scheme, and subgraphs from the same sub-tree are very close to each other in terms of their geometrical structure. Because these methods consider each subgraph independently, the selected subgraphs may have high redundancy, which imposes a great challenge in determining the optimal $K$ subgraphs for graph classification and also causes fluctuating results when the $K$ values are varied.

In contrast, the subgraph correlations for gBoost and RLMD are much smaller. 
The subgraphs discovered by gBoost and RLMD are highly overlapping (the first 5 subgraphs are identical). As pointed out by [21], $\ell_{1}$ regularization tends to select only one subgraph from a group of features and is not selective about which one is included, thus the redundancy among the features in gBoost is minimal. By using elastic norm, RLMD retains several group effects (some discriminative features may be included and excluded simultaneously), and usually achieves better results. This result is consistent with observations reported in [21] for vector data.

\subsubsection{Results on PTC Tasks}

We also conducted extensive experiments on the PTC datasets. The accuracies and AUC values (i.e., the area under ROC curves) are reported in Tables 3 and 4, where the results are obtained after RLMD converges, and $K=200$ for all filter methods.

Table 3: Accuracies and standard errors on PTC graphs with Optimal $K$ for 10 -fold cross-validation.

\begin{tabular}{|c|c|c|c|c|c|c|}
\hline ID & RLMD & $\begin{array}{c}\text { TOP } \\
+\mathrm{SVM}\end{array}$ & $\begin{array}{c}\text { IG } \\
+\mathrm{SVM}\end{array}$ & $\begin{array}{l}\text { gHSIC } \\
+ \text { SVM }\end{array}$ & $\begin{array}{l}\text { gSemi } \\
+ \text { SVM }\end{array}$ & gBoost \\
\hline MR & $\mathbf{0 . 6 5 5} \pm 0.013$ & $0.606 \pm 0.024$ & $0.607 \pm 0.025$ & $0.596 \pm 0.019$ & $0.601 \pm 0.020$ & $0.608 \pm 0.027$ \\
\hline MM & $\mathbf{0 . 6 6 4} \pm 0.019$ & $0.060_{ \pm 0.025}$ & $0.599_{ \pm 0.023}$ & $0.613 \pm 0.021$ & $0.603 \pm 0.019$ & $0.622 \pm 0.018$ \\
\hline FR & $\mathbf{0 . 7 0 4} \pm 0.018$ & $0.607 \pm 0.029$ & $0.584 \pm 0.029$ & $0.635 \pm 0.026$ & $0.619_{ \pm 0.023}$ & $0.678 \pm 0.008$ \\
\hline FM & $\mathbf{0 . 6 1 5} \pm 0.018$ & $0.592 \pm 0.031$ & $0.594 \pm 0.026$ & $0.581 \pm 0.021$ & $0.575 \pm 0.018$ & $0.603 \pm 0.017$ \\
\hline
\end{tabular}

Table 4: AUC values and standard errors on PTC graphs with Optimal $K$ for 10-fold cross-validation.

\begin{tabular}{|c|c|c|c|c|c|c|}
\hline ID & RLMD & $\begin{array}{c}\text { TOP } \\
+ \text { SVM }\end{array}$ & $\begin{array}{c}\text { IG } \\
+\mathrm{SVM}\end{array}$ & $\begin{array}{l}\text { gHSIC } \\
+ \text { SVM }\end{array}$ & $\begin{array}{l}\text { gSemi } \\
+ \text { SVM }\end{array}$ & gBoost \\
\hline MR & $\mathbf{0 . 6 8 1} \pm 0.021$ & $0.597 \pm 0.025$ & $0.596 \pm 0.025$ & $0.560 \pm 0.021$ & $0.580 \pm 0.021$ & $0.649 \pm 0.033$ \\
\hline MM & $\mathbf{0 . 6 8 0} \pm 0.020$ & $0.593 \pm 0.025$ & $0.590 \pm 0.025$ & $0.583 \pm 0.024$ & $0.600 \pm 0.022$ & $0.600_{ \pm 0.034}$ \\
\hline FR & $\mathbf{0 . 6 7 3} \pm 0.022$ & $0.600 \pm 0.029$ & $0.575 \pm 0.029$ & $0.618 \pm 0.021$ & $0.623 \pm 0.021$ & $0.640 \pm 0.026$ \\
\hline FM & $\mathbf{0 . 6 1 4} \pm 0.013$ & $0.585 \pm 0.032$ & $0.587 \pm 0.024$ & $0.582 \pm 0.021$ & $0.580 \pm 0.017$ & $0.583 \pm 0.017$ \\
\hline
\end{tabular}

The results in Tables 3 and 4 show that RLMD achieves considerable performance gains over all filter methods (TFM and DFM) and gBoost algorithm for all PTC datasets. 
Note that for PTC classifications, AUC values are more important because they are all imbalanced classification tasks.

\subsubsection{Parameter Analysis}

In this subsection, we study the impact of parameters $\gamma_{1}$ and $\gamma_{2}$ on algorithm performance. Both $\gamma_{1}$ and $\gamma_{2}$ values are selected from $\{0,0.01,0.03,0.05,0.07,0.09$, $0.11,0.15,0.2\}$, and the results under 10 -fold cross-validation on NCI-1 and NCI-33 are shown in Fig. 5.

Impact of $\gamma_{1}$ values: The experimental results in Fig. 5 show that $\gamma_{1}$ plays a more important role for the final classification model. With the increase of $\gamma_{1}$ from 0 to 0.2 , the classification performance drops rapidly in terms of accuracy.

Table 5: Impact of different $\gamma_{1}$ values on NCI-1 dataset with $\gamma_{2}=0.03, S_{\max }=200$.

\begin{tabular}{cccccc}
\hline$\gamma_{1}$ & 0 & 0.01 & 0.03 & 0.05 & 0.15 \\
\hline \#Selected Subgraphs & 200 & 180 & 130 & 65 & 0 \\
Accuracy & 0.775 & 0.759 & 0.711 & 0.679 & 0.5 \\
AUC & 0.831 & 0.811 & 0.795 & 0.713 & 0.5 \\
\hline
\end{tabular}

To better understand the impact of $\gamma_{1}$, we also summarize the number of subgraphs selected with different $\gamma_{1}$ values in Table 5. The results show that increasing $\gamma_{1}$ values will result in fewer subgraphs being selected for the final classifier model, because a larger $\ell_{1}$ norm regularizes more elements as 0 . For $\gamma_{1}=0$, there is no sparse solution. In other words, every subgraph should be used for graph classification. In this case, RLMD will only terminate when all subgraphs are incorporated for learning the model, or the maximum number of iterations $S_{\max }$ is reached. As the subgraph space is exponentially large, it is impractical to use all subgraph features to learn the model. The algorithm relies on $S_{\max }$ to terminate (200 is set in our experiment). The result shows that $\gamma_{1}=0$ even achieves better classification result, which is attributed to the fact that although $\ell_{1}$ regularization introduces a sparse solution, it may be biased in some applications [45], so the accuracy may drop. For other cases with $\gamma_{1}$ being considerably large $\left(\gamma_{1}=0.5\right)$, the regularization term dominates the objective function Eq.(4) with no subgraph being used for classification, which results in poor classification accuracy. 

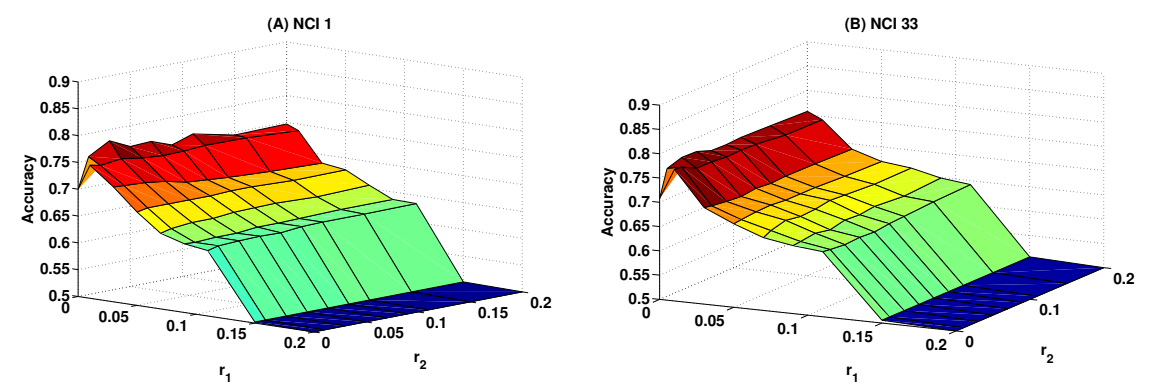

Figure 5: The accuracies with different $\gamma_{1}$ and $\gamma_{2}$ values.
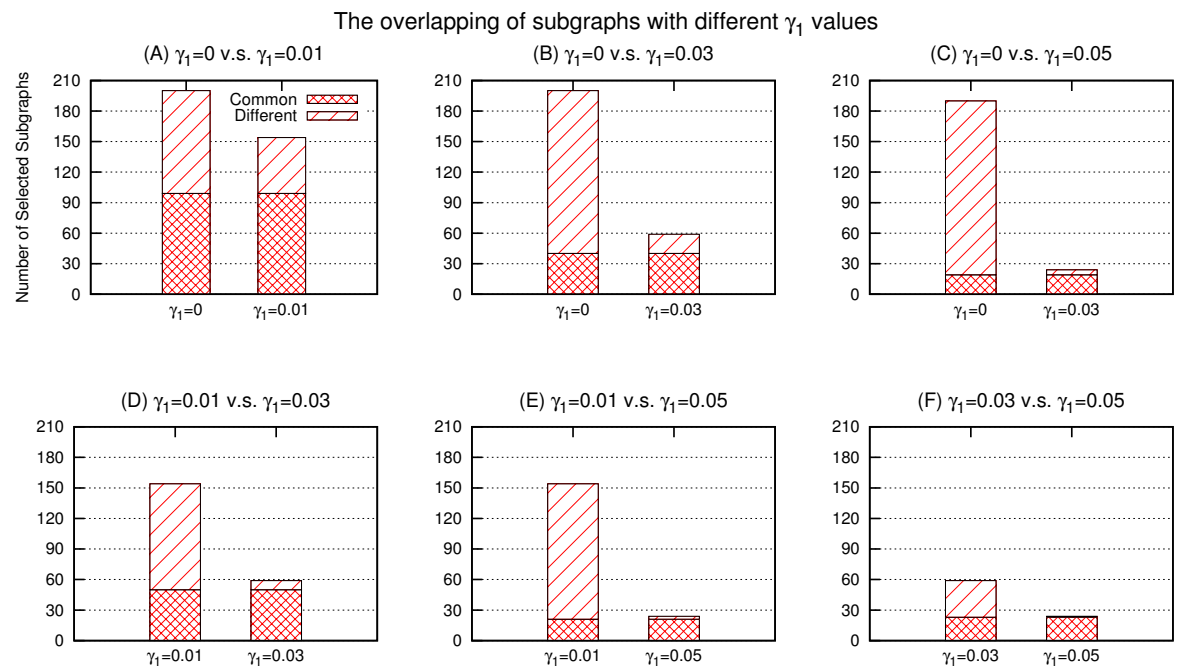

Figure 6: The overlapping of subgraphs (common subgraphs $v s$. different subgraphs) for different $\gamma_{1}$ values on NCI-1 dataset with a 70\%-30\% splitting on the NCI-1 dataset, i.e., 70\% graphs are randomly selected as training graphs, and $30 \%$ are used as test graphs. $\gamma_{2}=0.03$ and $S_{\max }=200$.

Table 6: Impact of different $\gamma_{2}$ values on NCI-1 dataset with $\gamma_{1}=0.01$.

\begin{tabular}{cccccc}
\hline$\gamma_{2}$ & 0 & 0.03 & 0.07 & 0.15 & 0.2 \\
\hline Accuracy & 0.748 & 0.759 & 0.74 & 0.741 & 0.72 \\
AUC & 0.810 & 0.811 & 0.806 & 0.811 & 0.78 \\
\hline
\end{tabular}


Table 7: Impacts of different $\epsilon$ values on NCI-1 dataset.

\begin{tabular}{ccccc}
\hline$\epsilon$ & 0.01 & 0.005 & 0.001 & 0.0001 \\
\hline Accuracy & 0.748 & 0.759 & 0.761 & 0.760 \\
AUC & 0.803 & 0.811 & 0.814 & 0.812 \\
\hline
\end{tabular}

Note that the convergence property of our algorithm is dependent on $\gamma_{1}$. In our experiments, we notice that $\gamma_{1}$ is very easy to set (in a small range [0.01,0.03]) for obtaining satisfactory results. This is much easier than requiring users to specify the number of subgraph features $K$ needed for each graph dataset, because users may not have any prior knowledge about the selection of $K$ values for different datasets, and different $K$ values often result in significant changes in the algorithm performance.

Interplay between $\gamma_{1}$ and subgraph selection: We further compare the common subgraphs selected by different $\gamma_{1}$ values, and report the results in Fig. 6. The results show that the subgraphs selected by using a smaller $\gamma_{1}$ values contain many subgraphs which are selected by using a larger $\gamma_{1}$ value. This observation is further evident in Fig.6.(E) and $(\mathrm{F})$. The reason is that a smaller $\gamma_{1}$ value will result in more subgraph features to be selected, which increases the possibility of covering a small subgraph set selected by using a larger $\gamma_{1}$ value. In other words, a slightly smaller $\gamma_{1}$ value will result in more subgraph feature candidates to be explored and be beneficial for the classification task. Impact of $\gamma_{2}$ values: We also vary $\gamma_{2}$ from 0 to 0.2 , and report the results in Table 6. The results show that a small regularization value $\gamma_{2}=0.03$ outperforms the case of $\gamma_{2}=0$, where the $\ell_{2}$ regularization effect disappears (only $\ell_{1}$ is used). This result is consistent with observations from a previous study [21]. This may be because $\ell_{1}$ ignores the correlated subgraphs in a group of features. When $\gamma_{2}$ keeps increasing, the classification performance drops because the larger $\ell_{2}$ regularization dominates the objective function and the loss minimization term has less effect.

Impact of $\epsilon$ values: We vary the $\epsilon$ values from 0.01 to 0.0001 to study the final classification performance of our algorithm, and report the final classification results in Table 7. The results show that as long as $\epsilon$ is subtle (from 0.001 to 0.0001 ), our classification can achieve similar classification results, i.e., an $\epsilon$-tolerance accuracy result to the 
optimal solution.

\section{Conclusion}

In this paper, we proposed a regularized loss minimization-driven (RLMD) graph classification method. We argued that existing filter-based subgraph selection methods simply focus on finding most discriminative subgraph features, and suffer severe disadvantages in determining the optimal number of subgraphs for graph classification and separating feature selection from the model learning phase. As a result, they might be able to find most discriminative subgraph features, but cannot form high accuracy classifiers because they cannot determine how many discriminative features are needed to train classifiers with the best performance gain. By integrating subgraph mining, discriminative subgraph selection, and model learning into one unified framework, RMLD is able to automatically determine the optimal number of discriminative subgraphs for best graph classification results. Our algorithm generalizes the state-of-the-art gBoost algorithm in the sense that it can employ any differentiable loss function and achieve better classification accuracy by using an elastic net regularization. Experimental results on real-world graph datasets show a clear performance gain over existing two-step filter methods (TFMs), direct filter methods (DFMs), and embedding methods.

\section{Acknowledgment}

We thank anonymous reviewers for their constructive comments. This work is partially supported by Australia ARC Discovery Project (DP140102206), and National Scholarship for Building High Level Universities, China Scholarship Council (No. 2011630030).

\section{References}

[1] M. Deshpande, M. Kuramochi, N. Wale, G. Karypis, Frequent substructure-based approaches for classifying chemical compounds, IEEE Transactions on Knowledge and Data Engineering (2005) 1036-1050. 
[2] J. Vogelstein, W. Roncal, R. Vogelstein, C. Priebe, Graph classification using signalsubgraphs: Applications in statistical connectomics, IEEE Transactions on Pattern Analysis and Machine Intelligence 35 (7) (2013) 1539-1551.

[3] A. Morales-Gonzlez, N. Acosta-Mendoza, A. Gago-Alonso, E. B. Garca-Reyes, J. E. Medina-Pagola, A new proposal for graph-based image classification using frequent approximate subgraphs, Pattern Recognition 47 (1) (2014) 169 - 177.

[4] S. Pan, X. Zhu, C. Zhang, P. S. Yu, Graph stream classification using labeled and unlabeled graphs, in: 2013 IEEE 29th International Conference on Data Engineering (ICDE), IEEE, 2013, pp. 398-409.

[5] F. Wysotzki, W. Kolbe, J. Selbig, Concept learning by structured examples-an algebraic approach., in: International Joint Conferences on Artificial Intelligence, 1981, pp. 153158.

[6] P. Geibel, F. Wysotzki, Learning relational concepts with decision trees, in: International Conference on Machine Learning, Vol. 96, 1996, pp. 166-174.

[7] B. J. Jain, K. Obermayer, Structure spaces, Journal of Machine Learning Research 10 (2009) 2667-2714.

[8] B. J. Jain, K. Obermayer, Learning in Riemannian orbifolds, arXiv preprint arXiv:1204.4294 (2012).

[9] H. Kashima, K. Tsuda, A. Inokuchi, Kernels for graphs, MIT Press, Cambridge (Massachusetts), 2004, Ch. In: Schlkopf B, Tsuda K, Vert JP, editors. Kernel methods in computational biology, pp. 101-113.

[10] H. Fei, J. Huan, Boosting with structure information in the functional space: an application to graph classification, in: ACM SIGKDD Conference on Knowledge Discovery and Data Mining (KDD), Washington DC, USA, 2010.

[11] X. Kong, P. Yu, Semi-supervised feature selection for graph classification, in: Proceedings of the 16th ACM SIGKDD international conference on Knowledge discovery and data mining, 2010, pp. 793-802.

[12] X. Kong, P. S. Yu, Multi-label feature selection for graph classification, in: IEEE International Conference on Data Mining, IEEE, 2010, pp. 274-283. 
[13] Y. Zhu, J. Yu, H. Cheng, L. Qin, Graph classification: A diversified discriminative feature selection approach, in: Conference on Information and Knowledge Management (CIKM), ACM, 2012, pp. 205-214.

[14] N. Jin, C. Young, W. Wang, Graph classification based on pattern co-occurrence, in: Conference on Information and Knowledge Management (CIKM), 2009, pp. 573-582.

[15] M. Thoma, H. Cheng, A. Gretton, J. Han, H. Kriegel, A. Smola, L. Song, P. Yu, X. Yan, K. Borgwardt, Near-optimal supervised feature selection among frequent subgraphs, in: SIAM International Conference on Data Mining, USA, 2009.

[16] S. Ranu, A. K. Singh, Graphsig: A scalable approach to mining significant subgraphs in large graph databases, in: International Conference on Data Engineering (ICDE), IEEE, 2009, pp. 844-855.

[17] X. Yan, H. Cheng, J. Han, P. S. Yu, Mining significant graph patterns by leap search, in: ACM SIGMOD Conference, ACM, 2008, pp. 433-444.

[18] H. Cheng, X. Yan, J. Han, Discriminative frequent pattern-based graph classification, Link Mining: Models, Algorithms, and Applications (2010) 237-262.

[19] I. Guyon, A. Elisseeff, An introduction to variable and feature selection, Journal of Machine Learning Research 3 (2003) 1157-1182.

[20] R. Tibshirani, Regression shrinkage and selection via the lasso, Journal of the Royal Statistical Society. Series B (Methodological) (1996) 267-288.

[21] H. Zou, T. Hastie, Regularization and variable selection via the elastic net, Journal of the Royal Statistical Society: Series B (Statistical Methodology) 67 (2) (2005) 301-320.

[22] T. Zhou, D. Tao, X. Wu, Manifold elastic net: A unified framework for sparse dimension reduction, Data Mining and Knowledge Discovery 22 (3) (2011) 340-371.

[23] H. Saigo, S. Nowozin, T. Kadowaki, T. Kudo, K. Tsuda, gBoost: A mathematical programming approach to graph classification and regression, Machine Learning 75 (2009) 69-89.

[24] M. Culp, G. Michailidis, Graph-based semisupervised learning, IEEE Transactions on Pattern Analysis and Machine Intelligence 30 (1) (2008) 174-179. 
[25] A. Mantrach, N. van Zeebroeck, P. Francq, M. Shimbo, H. Bersini, M. Saerens, Semisupervised classification and betweenness computation on large, sparse, directed graphs, Pattern Recognition 44 (6) (2011) 1212 - 1224.

[26] M. Karasuyama, H. Mamitsuka, Multiple graph label propagation by sparse integration, IEEE Transactions on Neural Networks and Learning Systems 24 (12) (2013) 1999-2012.

[27] X. Liu, L. Wang, J. Zhang, J. Yin, H. Liu, Global and local structure preservation for feature selection, IEEE Transactions on Neural Networks and Learning Systems 25 (6) (2014) 1083-1095.

[28] D. Liben-Nowell, J. Kleinberg, The link-prediction problem for social networks, Journal of the American Society for Information Science and Technology 58 (7) (2007) 1019-1031.

[29] C. Nguyen, H. Mamitsuka, Latent feature kernels for link prediction on sparse graphs, IEEE Transactions on Neural Networks and Learning Systems 23 (11) (2012) 1793-1804.

[30] N. Jin, C. Young, W. Wang, GAIA: graph classification using evolutionary computation, in: ACM SIGMOD Conference, ACM, 2010, pp. 879-890.

[31] J. Wu, X. Zhu, C. Zhang, P. Yu, Bag constrained structure pattern mining for multi-graph classification, IEEE Transactions on Knowledge and Data Engineering 26 (10) (2014) 2382 -2396 .

[32] L. Bai, L. Rossi, A. Torsello, E. R. Hancock, A quantum Jensen-Shannon graph kernel for unattributed graphs, Pattern Recognition 48 (2) (2015) 344 - 355.

[33] N. Shervashidze, P. Schweitzer, E. J. Van Leeuwen, K. Mehlhorn, K. M. Borgwardt, Weisfeiler-Lehman graph kernels, Journal of Machine Learning Research 12 (2011) 2539_ 2561.

[34] M. Neumann, N. Patricia, R. Garnett, K. Kersting, Efficient graph kernels by randomization, in: Machine Learning and Knowledge Discovery in Databases, Springer, 2012, pp. 378-393.

[35] K. Riesen, H. Bunke, Graph classification by means of Lipschitz embedding, IEEE Transactions on SMC, Part B: Cybernetics 39 (2009) 1472-1483.

[36] J. Wu, Z. Hong, S. Pan, X. Zhu, C. Zhang, Multi-graph-view learning for graph classification, in: IEEE International Conference on Data Mining, 2014, pp. 590-599. 
[37] J. Wu, S. Pan, X. Zhu, Z. Cai, Boosting for multi-graph classification, IEEE Transactions on Cybernetics 45 (3) (2015) 430-443.

[38] J. Wu, Z. Hong, S. Pan, X. Zhu, C. Zhang, Z. Cai, Multi-graph learning with positive and unlabeled bags, in: SIAM International Conference on Data Mining, SIAM, 2014, pp. $217-225$.

[39] S. Pan, X. Zhu, Graph classification with imbalanced class distributions and noise, in: International Joint Conferences on Artificial Intelligence, 2013, pp. 1586-1592.

[40] S. Pan, J. Wu, X. Zhu, C. Zhang, Graph ensemble boosting for imbalanced noisy graph stream classification, IEEE Transactions on Cybernetics 45 (5) (2015) 940-954.

[41] S. Pan, J. Wu, X. Zhu, Cogboost: Boosting for fast cost-sensitive graph classification, IEEE Transactions on Knowledge and Data Engineering (In Press) (2015) 1. doi:10.1109/ TKDE.2015.2391115.

[42] X. Yan, J. Han, gSpan: Graph-based substructure pattern mining, in: IEEE International Conference on Data Mining, 2002, pp. 721-724.

[43] A. Demiriz, K. Bennett, J. Shawe-Taylor, Linear programming boosting via column generation, Machine Learning (2002) 225-254.

[44] T. Kudo, E. Maeda, Y. Matsumoto, An application of boosting to graph classification, in: Neural Information Processing Systems (NIPS), 2004, pp. 729-736.

[45] M. Tan, I. W. Tsang, L. Wang, Towards ultrahigh dimensional feature selection for big data, Journal of Machine Learning Research 15 (2014) 1371-1429. 